\title{
Constructing Realistic Canine Bilayer Biatrial Mesh for the Modeling and Simulation of Atria Fibrillation
}

\author{
Mirabeau Saha ${ }^{1,2}$, Caroline Roney ${ }^{3}$, Feng Xiong ${ }^{1}$, Hubert Cochet $^{2}$, Stephanie Tan ${ }^{1}$, Steven Niederer ${ }^{3}$, \\ Edward Vigmond ${ }^{2}$, and Stanley Nattel ${ }^{1,2,4}$ \\ ${ }^{1}$ Department of Medicine and Research Center, Montreal Heart Institute, Montreal, QC, Canada \\ ${ }^{2}$ IHU Liryc, Electrophysiology and Heart Modeling Institute, Fondation Bordeaux Université, Pessac- \\ Bordeaux, France \\ ${ }^{3}$ Department of Biomedical Engineering, King's College London, London, United Kingdom \\ ${ }^{4}$ Institute of Pharmacology, West German Heart and Vascular Center, University Duisburg-Essen, \\ Essen, Germany
}

\begin{abstract}
An improved appreciation of the mechanisms underlying atrial fibrillation $(A F)$ is essential for better arrhythmia management. A deeper understanding requires a full consideration of atrial geometry. Here, we develop the first anatomically accurate high-resolution canine atrial computational bilayer model for AF studies.

Canine CT-scan imaging data were segmented and used to reconstruct the Bachmann's bundle (BB), the left atrium (LA) and right atrium (RA). The LA is dilated to obtain the second layer. The $R A$ endocardial layer consists of the sinus node ( $S A N)$, the pectinate muscles (PMs) and the crista terminalis (CT). The Ramirez-Nattel-Courtemanche canine cell model was used to simulate electrical activity. Activation time (AT) and action potential duration (APD) were computed. The obtained bilayer mesh has high resolution with average edge length $276 \pm 58 \mu \mathrm{m}$. Action potential propagation from the $S A N$ was realistic and its path along CS presumes an important role of the CS in the initiation and maintenance of rotors during $A F$. The propagation time from SAN to PVS was $\sim 119 \mathrm{~ms}$ and $A P D 90$ was heterogeneous in the model.

This new bilayer model with realistic geometry, combined with experimental data, will help to better understand $A F$ and its underlying mechanisms, in order to develop better prognostic and therapeutic tools.
\end{abstract}

\section{Introduction}

Atrial fibrillation (AF) is the most common disorder of cardiac rhythm. The atrial remodelling induced by underlying conditions and risk factors, as well as by $\mathrm{AF}$ itself, is an important factor in the natural history of the disease [1]. However, the mechanisms causing this remodelling and mediating its profibrillatory consequences, whether electrophysiological or structural [2], remain poorly understood. Understanding these mechanisms is essential to improve the diagnostic and therapeutic management of patients with AF. That understanding requires full and detailed consideration of the geometry of the atria. Three dimensional mathematical models of the atria are increasingly used because controlling factors can be modified individually or in combination with a high degree of precision. Canine AF exhibits many properties similar to human. To obtain geometrically realistic mathematical models, it is necessary to obtain data from in vivo medical imaging. CTScan is a modality that is suitable for canine atria in detail $[3,4]$. Human bilayer biatrial model has been recently successfully used for AF studies [5]. Our laboratory is carrying out numerous AF clinical studies on canine atria. In this study, we have constructed a bilayer biatrial model for canine atria, providing a more detailed and more realistic 3D mathematical model of the canine atria geometry for AF studies and the underlying mechanisms. Bilayer 3D models are a compromise between monolayer and three-dimensional models.

\section{Methods}

\section{$2.1 \quad$ CT-Scan Imaging process}

A $24.8 \mathrm{Kg}$ 5-year-old dog was used in this study approved by the Institutional Animal Research Ethics Committee at the Montreal Heart Institute. The animal was sedated and ventilated with $100 \% \mathrm{FiO}_{2}$. Intravenous 
access was obtained with an 18-gauge catheter placed in a femoral vein. The beta-blocker metoprolol ( $5 \mathrm{mg}$ IV q 5 minutes; maximum dose of $15 \mathrm{mg}$ ) was given to achieve a heart rate of $<130$ beats-per-minute.

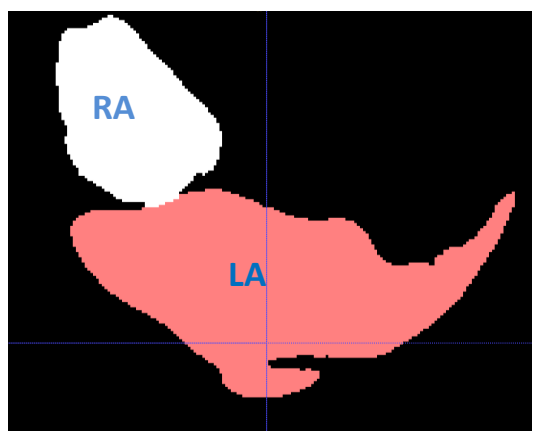

Figure 1: An image slice with the left atrium (LA) segmentation shown in red and right atrium (RA) in white.

The computed-tomography scan (CT-Scan) was performed with a 128-section dual-source CT system (Somatom Definition Flash; Siemens Medical Solutions, Forchheim, Germany). Images were obtained using an ECG-triggered retrospective axial acquisition from $0 \%$ $100 \%$ of the $\mathrm{R}-\mathrm{R}$ interval (from the $\mathrm{R}$ wave to the next $\mathrm{R}$ wave) with a high-pitch and $\mathrm{z}$-axis centered on the heart. CT parameters were as follows: collimation, $2 \times 64.0 \times 0.6$ $\mathrm{mm}$; gantry rotation time, $280 \mathrm{~ms}$; temporal resolution, 75 $\mathrm{ms}$; voltage of both tubes, $120 \mathrm{kV}$; tube current, $500 \mathrm{mAs}$ for the arterial phase and $450 \mathrm{mAs}$ for the late phase. Ventilation was momentarily suspended for image acquisition. Forty-eight milliliters of nonionic, lowismolar contrast medium (opamidol, Isovue [370 mg iodine per $\mathrm{mL}$ ]; Bracco) was injected at a rate of $4 \mathrm{~mL} / \mathrm{s}$, followed by $10 \mathrm{~mL}$ of a 50/50 mix of contrast and isotonic saline solution. Bolus tracking was used with a region of interest placed in the descending aorta and trigger set at $250 \mathrm{HU}$ (Houndsfield Unit) and the late phase was acquired immediately thereafter. Reconstruction of both phases was performed at $5 \%$ of the R-R interval field of view limited to the heart with matrix size of $512 \times 512$ pixels, section thickness $0.75 \mathrm{~mm}$ and $0.3 \mathrm{~mm}$ increment.

\subsection{Atrial structure extraction, processing and recombination}

To remove non atrial structures from DICOM data, volume rendering reconstructions were perormed as shown in Fig. 1. Then, with the same protocol we applied to obtain a human patient-specific bilayer biatrial models [6], the RA epicardial layer, LA and Bachmann's bundle (BB) endocardial surfaces were obtained with manual segmentation using ITK-Snap tool. The RA included a long coronary sinus (CS). Each structure was then meshed and smoothed with mcubes tool and trimmed using paraview software. The Fossa Ovalis (FO) position was identified on each atrium. RA structures like the sinoatrial node (SAN), the pectinate muscles (PMs) and the crista terminalis (CT) were computed from connection points and included in the RA as an endocardial layer. Fedorov et al. [7] found experimentally a zone of conduction block near the SAN, which we implemented in this bilayer model. The LA endocardial layer is dilated to construct the LA epicardial layer.

Fiber directions were assigned to each part of the model using the approach we implemented previously [8]. Figure 2 shows fiber orientation in various structures. BB and $\mathrm{FO}$ were designed to connect RA to LA manually with additional triangular elements. The RA was also manually connected to the LA through the CS with triangular elements. The LA layers were joined to each other by discrete linear electrical connections.
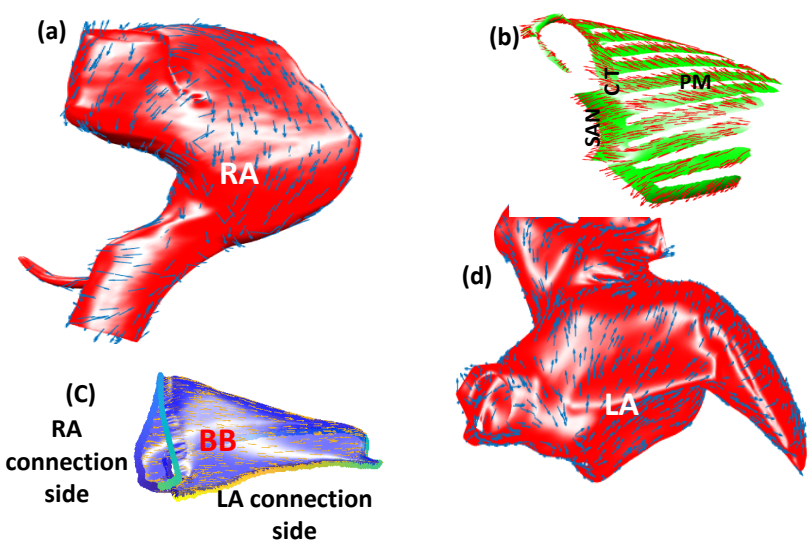

Figure 2: Atrial structures with fiber directions. For viewing purposes, 1/100 fibers represented on RA (a), 1/5 on SAN, CT and PMs (b), 1/20 on BB (c) and 1/100 on bilayer LA (d)

\subsection{Electro-physiological simulation}

To test the model, the Ramirez-Nattel-Courtemanche canine cell model [9] was used to simulate cellular electrical activity. The monodomain equation was used to describe electrical propagation and solved with the use of the finite element method. Ionic properties and conductances were adjusted to achieve realistic propagation speeds as illustrated in Table 1 motivated by previous studies $[6,10]$. A passive ionic model was used in the inferior vena cava (IVC) and the regions of conduction block, with the resting potential set at $-83 \mathrm{mV}$. Sinus-node activation was simulated by stimulating the SAN nodes with $27 \mathrm{mV}$ stimuli at $1.67 \mathrm{~Hz}$ and the activation time (AT) to the pulmonary veins (PVs) and LA appendage (LAA) was measured. The action potential duration at $90 \%$ repolarization $\left(\mathrm{APD}_{90}\right)$ was computed on each node of the mesh 
Table 1: Ionic and fiber properties

\begin{tabular}{|c|c|c|c|c|c|c|c|c|c|c|c|}
\hline & & RA & RAA & LA & LAA & PVs & PM & CT & BB & SAN & $\begin{array}{r}\text { IVC et } \\
\text { Block }\end{array}$ \\
\hline \multirow{6}{*}{ 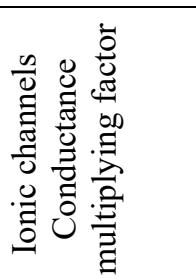 } & $\mathrm{g}_{\text {cal }}$ & 0.3 & 0.32 & 0.3 & 0.32 & 0.25 & 0.3 & 0.5 & 0.5 & 1.0 & 0.3 \\
\hline & $\mathrm{g}_{\mathrm{t} 0}$ & 0.34 & 0.44 & 0.34 & 0.44 & 0.32 & 0.18 & 0.64 & 0.64 & 1.0 & 0.34 \\
\hline & $\mathrm{gkr}_{\mathrm{kr}}$ & 1.0 & 1.0 & 1.6 & 1.6 & 2.4 & 1.0 & 1.0 & 1.0 & 1.0 & 1.0 \\
\hline & $\mathrm{g}_{\text {kur }}$ & 0.5 & 0.5 & 0.5 & 0.5 & 0.35 & 0.4 & 0.5 & 0.5 & 0.5 & 0.5 \\
\hline & $\mathrm{g}_{\mathrm{k} 1}$ & 1.0 & 1,12 & 1.0 & 1.12 & 1.1 & 1.0 & 1.0 & 1.0 & 1.6 & 1.0 \\
\hline & $\mathrm{g}_{\mathrm{ks}}$ & 1.0 & 1.0 & 1.0 & 1.0 & 1.87 & 1.0 & 1.0 & 1.0 & 1.0 & 1.0 \\
\hline \multirow{2}{*}{$\begin{array}{c}\text { Fiber } \\
\text { conductivity }\end{array}$} & Longitudinal & 3.8 & 3.8 & 3.8 & 3.8 & 3.8 & 2.8 & 1.97 & 17.3 & 1.57 & 0.0 \\
\hline & Transversal & 1.02 & 1.02 & 1.02 & 1.02 & 1.02 & 0.12 & 0.3 & 12.4 & 1.57 & 0.0 \\
\hline
\end{tabular}

\section{Results and Discussion}

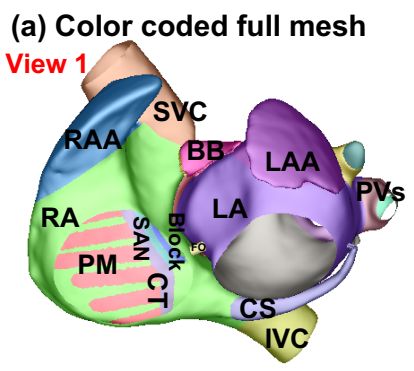

(b) Fibers in the full mesh
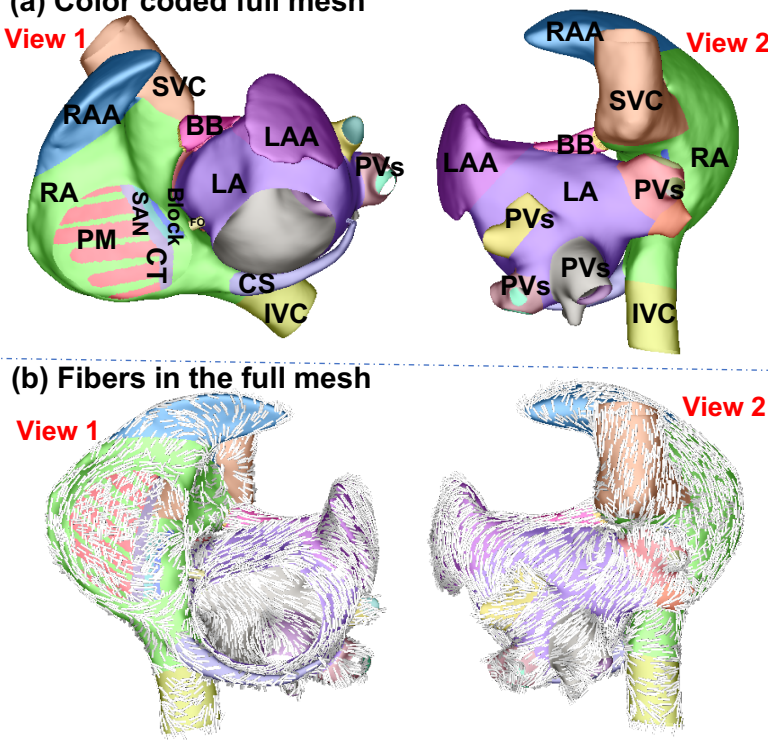

Figure 3: (a) Canine specific bilayer atria model with regions colors coded. (b) Fiber directions in the mesh.

Figure 3(a), with regions colors coded, represents the resulting canine-specific bilayer biatrial mesh. Figure 3(b) shows the full mesh including the fiber directions (where $1 / 100$ fibers are represented). It contains $1,179,133$ nodes and 2,346,395 triangular elements in total, 350,909 line elements to couple LA layers and 353,445 line elements in total. The average edge length is $276 \pm 58 \mu \mathrm{m}$. The model has the five PVs observed on CT-imaging. This model constitutes the first realistic canine bilayer biatrial mesh including a long realistic CS. Previous studies have suggested that the CS may play a role in perpetuating AF [11] and can be target for ablation therapy of permanent $\mathrm{AF}$ [12], but the role of the $\mathrm{CS}$ in $\mathrm{AF}$ initiation and perpetuation are not well understood. This new model will help to explore the role of the CS in AF.

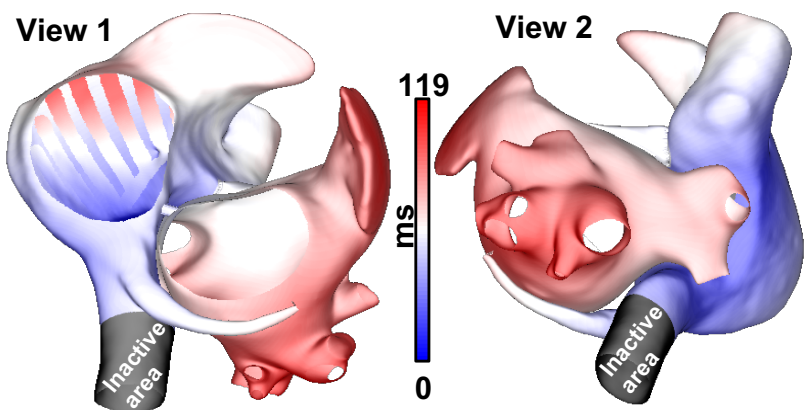

Figure 4: Activation time (AT) map showing propagation from SAN to PVs and LAA tip.

Electrical activity originating in the SAN travels around the zone of block, undergoing a delay. It continues through $\mathrm{BB}$ and FO to reach the LA. The total propagation time over the mesh, to reach the last node on PVs, was $\sim 119 \mathrm{~ms}$, as shown in Fig.4. As illustrated in Fig. 5, APD 90 was $\sim 108$ $\mathrm{ms}$ on the PVs, $\sim 128 \mathrm{~ms}$ on LAA, $\sim 134 \mathrm{~ms}$ on LA, $\sim 139$ ms on RAA, $\sim 146 \mathrm{~ms}$ on RA, $\sim 196 \mathrm{~ms}$ on $\mathrm{BB}$ and $\mathrm{CT}$, $\sim 158 \mathrm{~ms}$ on PM and $\sim 235.81 \mathrm{~ms}$ on SAN. These results are in agreement with experimental measurements $[9,10]$

It is known that intrinsic APD heterogeneity among atrial regions, as obtained in this study, can affect conduction in regions with very long refractoriness $[3,9]$ and play an important role in AF dynamics.

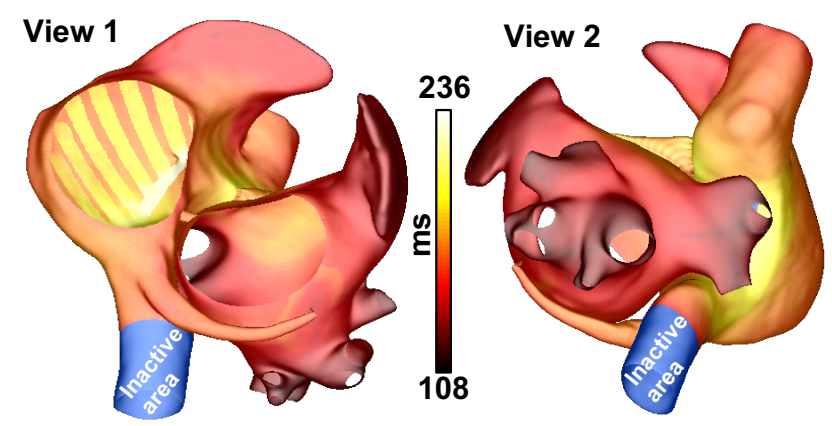

Figure 5: Action potential duration (APD90) map. 


\section{Conclusion}

In this work, we have built the first canine-specific realistic bilayer biatrial model. Since this model can significantly reduce computation times while retaining valuable features affecting propagation, it provides a good trade-off between the limited monolayer model and the volumetric model which imposes an important computational load. This model will help for investigating mechanisms determining AF dynamics as well as in understanding and testing various ablation strategies.

\section{Acknowledgments}

For this study, computer time was partially provided by the computing facilities Cedar on Compute Canada. The authors thank Nathalie L'Heureux for valuable technical support.

\section{References}

[1] Nattel S, Harada M. "Atrial remodeling and atrial fibrillation: recent advances and translational perspectives". J. of the Am. Col. of Card. 2014; 63(22):2335-2345.

[2] Schotten U, Verheule S, Kirchhof P, Goette A. Pathophysiological mechanisms of atrial fibrillation: a translational appraisal. Physiological Reviews 2011;91(1):265-325.

[3] Aslanidi OV, Nikolaidou T, Zhao J, Smaill BH, Gilbert SH, Holden AV, Lowe T, Withers PJ, Stephenson RS, Jarvis JC, et al. Application of micro-computed tomography with iodine staining to cardiac imaging, segmentation, and computational model development. IEEE Transactions on Medical Imaging 2012;32(1):8-17.

[4] Genain MA, Morlet A, Herrtage M, Muresian H, Anselme F, Latremouille C, Laborde F, Behr L, Borenstein N. Comparative anatomy and angiography of the cardiac coronary venous system in four species: human, ovine, porcine, and canine. Journal of Veterinary Cardiology 2018;20(1):33-44.

[5] Roney CH, Ng FS, Debney MT, Eichhorn C, Nachiappan A, Chowdhury RA, Qureshi NA, Cantwell CD, Tweedy JH, Niederer SA, et al. Determinants of new wavefront locations in cholinergic atrial fibrillation. EP Europace 2018; 20(suppl 3):iii3-iii15.

[6] Labarthe S, Bayer J, Coudière Y, Henry J, Cochet H, Jaïs P, Vigmond E. A bilayer model of human atria: mathematical background, construction, and assessment. Europace 2014; 16(suppl 4):iv21-iv29.

[7] Fedorov VV, Schuessler RB, Hemphill M, Ambrosi CM, Chang R, Voloshina AS, Brown K, Hucker WJ, Efimov IR. Structural and functional evidence for discrete exit pathways that connect the canine sinoatrial node and atria. Circulation Research 2009;104(7):915-923.

[8] Roney CH,Williams SE, Cochet H, Mukherjee RK, O’Neill L, Sim I, Whitaker J, Razeghi O, Klein GJ, Vigmond EJ, et al. Patient-specific simulations predict efficacy of ablation of interatrial connections for treatment of persistent atrial fibrillation. EP Europace 2018; 20:iii55-iii68.
[9] Ramirez RJ, Nattel S, Courtemanche M. Mathematical analysis of canine atrial action potentials: rate, regional factors, and electrical remodeling. American Journal of Physiology Heart and Circulatory Physiology 2000; 279(4):H1767-H1785.

[10] Ehrlich JR, Cha TJ, Zhang L, Chartier D, Melnyk P, Hohnloser SH, Nattel S. Cellular electrophysiology of canine pulmonary vein cardiomyocytes: action potential and ionic current properties. The Journal of Physiology 2003; 551(3):801-813.

[11] Oral H, Ozaydin M, Chugh A, Scharf C, Tada H, Hall B, Cheung P, PELOSI Jr F, Knight BP, Morady F. Role of the coronary sinus in maintenance of atrial fibrillation. Journal of Cardiovascular Electrophysiology 2003;14(12):1329-1336.

[12] Ahmed N, Rungatscher A, Linardi D, Molon G, Luciani GB, Faggian G. Pp-158 coronary sinus can be target for permanent atrial fibrillation ablation therapy? American Journal of Cardiology 2016;117:S98.

Address for correspondence:

Mirabeau Saha

Montreal Heart Institute, 5000 Rue Bélanger, S4501, Montreal, Quebec, H1T 1C8, Canada

mirabeau.saha@mail.mcgill.ca 